\title{
Combinatorial etude
}

\author{
Miroslav Stoenchev ${ }^{*}$, Venelin Todorov ${ }^{\dagger \ddagger}$ \\ * Technical University of Sofia, Bulgaria \\ $\dagger$ Institute of Mathematics and Informatics, Bulgarian Academy of Sciences \\ $\ddagger$ Institute of Information and Communication Technologies, Bulgarian Academy of Sciences \\ Email: mrs@tu-sofia.bg,vtodorov@math.bas.bg, venelin@parallel.bas.bg,
}

\begin{abstract}
The purpose of this article is to consider a special class of combinatorial problems, the so called Prouhet-TarryEscot problem, solution of which is realized by constructing finite sequences of \pm 1 . For example, for fixed $p \in \mathbb{N}$, is well known the existence of $n_{p} \in \mathbb{N}$ with the property: any set of $n_{p}$ consecutive integers can be divided into 2 sets, with equal sums of its $p^{\text {th }}$. powers. The considered property remains valid also for sets of finite arithmetic progressions of complex numbers.
\end{abstract}

\section{Morse SEQUenCE}

$\mathbf{F}$ OR every positive integer $m$, let us denote with $\vartheta(m)$ and $\varrho(m)$ respectively the number of occurences of digit 1 in the binary representation of $m$, and the position of first digit 1 in the binary representation of $m$. The Morse sequence $\left\{a_{m}\right\}_{m=1}^{\infty}$ ([1], [4]) is defined by

$$
a_{m}=(-1)^{\vartheta(m)+\varrho(m)-2} .
$$

The following properties are derived directly:

$$
\begin{gathered}
a_{2^{k}}=(-1)^{k} \\
a_{2^{k}+l}=-a_{l} \text { for } l=1,2 \ldots, 2^{k} .
\end{gathered}
$$

The problem of finding a number $n_{p}$, such that the set $A_{n_{p}}=\left\{1,2, \ldots, n_{p}\right\}$ is represented as disjoint union of two subsets, say $B$ and $C$, with the property:

$$
\sum_{b \in B} b^{p}=\sum_{c \in C} c^{p}
$$

is solved by the sequence $\left\{a_{m}\right\}_{m=1}^{\infty}$. Elementary proof is given below ${ }^{1}$ and $n_{p}=2^{p+1}$ has the desired property, with

$$
\begin{gathered}
B=\left\{m \in A_{n_{p}}: a_{m}=1\right\}, \\
C=A_{n_{p}} \backslash B=\left\{m \in A_{n_{p}}: a_{m}=-1\right\} .
\end{gathered}
$$

This result can be generalized to arbitrary arithmetic progressions of complex numbers. As example, if $a, d \in \mathbb{C}, d \neq 0$ and $A_{n_{p}}=\left\{a+k d: k=0,1, \ldots, n_{p}-1\right\}$, then $n_{p}=2^{p+1}$ and $B=\left\{a+k d \in A_{n_{p}}: a_{k+1}=1\right\}$.

\footnotetext{
${ }^{1}$ Similar solutions and generalizations of the Prouhet-Tarry-Escot problem are considered in [2], [3], [5], [6], [8]
}

\section{FORMULATION OF THE MAIN RESULTS}

Let us define $\left\{H_{n, m}(z)\right\}_{n, m=1}^{\infty}$, by

$$
H_{n, m}(z)=\sum_{l=n}^{\infty} \sum_{k=1}^{2^{l}} a_{k}(P(z)+k \cdot Q(z))^{m},
$$

where $P, Q \in \mathbb{C}[z]$ are complex polynomials.

Proposition 1: If $m=0,1, \ldots, n-1$ then $H_{n, m} \equiv 0$, while if $n$ is even number the following equality is satisfied

$$
H_{n, n}(z)=n ! 2^{\frac{n^{2}-n}{2}} Q^{n}(z) .
$$

Proposition 2: Let $n \in \mathbb{N}$ be a even number and $\alpha_{1}, \alpha_{2}, \ldots, \alpha_{n}$ are complex numbers, then

$$
\sum_{k=1}^{2^{n}} a_{k}\left(\alpha_{1}+k\right)\left(\alpha_{2}+k\right) \cdots\left(\alpha_{n}+k\right)=n ! 2^{\frac{n^{2}-n}{2}} .
$$

Proposition 3: If $P \in \mathbb{C}[z]$ is a complex polynomial, then

$$
\sum_{k=1}^{2^{1+\operatorname{deg} P}} a_{k} P(k)=0 .
$$

Proposition 4: Let $p$ and $k$ be positive integers. Then there exist $n \in \mathbb{N}, n \leq 2^{p\left\lceil\log _{2} k\right\rceil}$ and distinct square-free positive integers $x_{i j}, i=1,2, \ldots, k ; j=1,2, \ldots, n$ with the property:

$$
\sum_{j=1}^{n} x_{1 j}^{r}=\sum_{j=1}^{n} x_{2 j}^{r}=\cdots=\sum_{j=1}^{n} x_{k j}^{r}, \forall r=1,2, \ldots, p .
$$

Let $\left\{b_{i}\right\}_{i=0}^{\infty}$ and $\left\{d_{i}\right\}_{i=0}^{\infty}$ be arbitrary sequences of complex numbers. Let $\gamma_{n}$ and $\Gamma_{n}$ be the sets:

$$
\begin{gathered}
\gamma_{m}=\left\{b_{m}+k d_{m}: k \in \mathbb{Z}\right\} \text { and } \\
\Gamma_{n}=\gamma_{1} \gamma_{2} \ldots \gamma_{n}=\left\{\prod_{i=1}^{n}\left(b_{i}+k d_{i}\right): k \in \mathbb{Z}\right\} .
\end{gathered}
$$

Proposition 5: Let $n$ and $m$ be positve integers. Then there exists an integer $s=s(n, m)$ with the property: every $s$-element subset of $\Gamma_{n}$, where $k$ runs through $s$ consequtive integers, can be represented as disjoint union of $m$ subsets, with equal sums of the elements in each one.

The proof of each of the formulated above propositions, with the exception for 5 , is based on the following lemma: 
Lemma 1: Set $a, d \in \mathbb{C}, d \neq 0, p \in \mathbb{N}$ and $A_{2^{p+1}}=\{a+$ $\left.k d: k=0,1, \ldots, 2^{p+1}-1\right\}$. Then there are sets $B \cap C=\emptyset$, $B \cup C=A_{2^{p+1}}$ such that

$$
\sum_{b \in B} b^{p}=\sum_{c \in C} c^{p} .
$$

Corollary 1: Under assumptions of lemma 1, it holds

$$
\sum_{b \in B} b^{r}=\sum_{c \in C} c^{r}, r=0,1, \ldots, p .
$$

To prove lemma 1 and its consequence, we define a sequence of polynomials: $\left\{T_{s, p}(z)\right\}_{s=0}^{\infty}$, through which we will gradually calculate the differences between the sums of equal powers of the elements in $B=\left\{a+k d \in A_{2^{p+1}}: a_{k+1}=1\right\}$ and $C=\left\{a+k d \in A_{2^{p+1}}: a_{k+1}=-1\right\}$. For $s \geq 0$ set

$$
T_{s, p}(z)=\sum_{k=0}^{4^{s+1}-1} a_{k+1}(z+k d)^{p}
$$

and we calculate

$$
T_{s, p}(z)=
$$

$\sum_{0 \leq k \leq 4^{s+1}-1 ; a_{k+1}=1}(z+k d)^{p}-\sum_{0 \leq k \leq 4^{s+1}-1 ; a_{k+1}=-1}(z+k d)^{p}$.

When $s \leq \frac{p-1}{2}$, set $z=a$ to obtain

$$
T_{s, p}(a)=\sum_{b \leq a+\left(4^{s+1}-1\right) d} b^{p}-\sum_{c \leq a+\left(4^{s+1}-1\right) d} c^{p},
$$

where summation is by $b \in B, c \in C$.

Set $p=2 m+r, r \in\{0,1\}$. Here and everywhere below the summations are performed on all $b \in B$ and $c \in C$, which satisfy the corresponding inequalities.

When $r=1$ we obtain

$$
\begin{gathered}
T_{m, p}(a)=\sum_{b \leq a+\left(4^{m+1}-1\right) d} b^{p}-\sum_{c \leq a+\left(4^{m+1}-1\right) d} c^{p} \\
=\sum_{b \leq a+\left(2^{p+1}-1\right) d} b^{p}-\sum_{c \leq a+\left(2^{p+1}-1\right) d} c^{p} \\
=\sum_{b \in B} b^{p}-\sum_{c \in C} c^{p} .
\end{gathered}
$$

When $r=0$ :

$$
\begin{gathered}
T_{m-1, p}(a)=\sum_{b \leq a+\left(2^{2 m}-1\right) d} b^{p}-\sum_{c \leq a+\left(2^{2 m}-1\right) d} c^{p} \\
=\sum_{b \leq a+\left(2^{p}-1\right) d} b^{p}-\sum_{c \leq a+\left(2^{p}-1\right) d} c^{p} .
\end{gathered}
$$

On the other hand

$$
\begin{aligned}
& \sum_{a+2^{p} d \leq b \leq a+\left(2^{p+1}-1\right) d} b^{p}-\sum_{a+2^{p} d \leq c \leq a+\left(2^{p}-1\right) d} c^{p} \\
& =\sum_{2^{p} \leq m \leq 2^{p+1}-1} a_{m+1}(a+m d)^{p}
\end{aligned}
$$

$$
\begin{aligned}
& =\sum_{k=0}^{2^{p}-1} a_{2^{p}+k+1}\left(a+\left(2^{p}+k\right) d\right)^{p} \\
& =-\sum_{k=0}^{2^{p}-1} a_{k+1}\left(a+\left(2^{p}+k\right) d\right)^{p} \\
& =-\sum_{k=0}^{2^{p}-1} a_{k+1}\left(\left(a+2^{p} d\right)+k d\right)^{p}= \\
& =-\sum_{k=0}^{2^{2 m}-1} a_{k+1}\left(\left(a+2^{p} d\right)+k d\right)^{p} \\
& =-T_{m-1, p}\left(a+2^{p} d\right) .
\end{aligned}
$$

Therefore, for $p=2 m$ we obtain

$$
\begin{gathered}
\sum_{b \in B} b^{p}-\sum_{c \in C} c^{p}= \\
T_{m-1, p}(a)-T_{m-1, p}\left(a+2^{p} d\right) .
\end{gathered}
$$

Summarized:

$$
\sum_{b \in B} b^{p}-\sum_{c \in C} c^{p}=
$$

$\left\{\begin{array}{rll}T_{m, p}(a), & \text { for } & p=2 m+1 \\ T_{m-1, p}(a)-T_{m-1, p}\left(a+2^{p} d\right), & \text { for } & p=2 m\end{array}\right.$

\section{Proof OF THE MAIN RESUlts}

Lemma 1 follows directly from :

\section{Proposition 6:}

$$
T_{m-1, p}(z)=\left\{\begin{array}{rll}
0, & \text { when } & p=2 m-1 \\
p ! 2^{\frac{p^{2}-p}{2}} d^{p}, & \text { when } & p=2 m
\end{array}\right.
$$

Proof 1: Let us determine the polynomials $\left\{T_{s, p}(z)\right\}_{s=0}^{\infty}$ by finding recurrent formula. Since $a_{1}=a_{4}=1, a_{2}=a_{3}=-1$, then

$$
T_{0, p}(z)=(z+3 d)^{p}-(z+2 d)^{p}-(z+d)^{p}+z^{p} .
$$

We will prove that for all $s \geq 1$ is valid

$$
\begin{aligned}
T_{s, p}(z)= & T_{s-1, p}\left(z+3.4^{s} d\right)-T_{s-1, p}\left(z+2.4^{s} d\right) \\
& -T_{s-1, p}\left(z+4^{s} d\right)+T_{s-1, p}(z) .
\end{aligned}
$$

For example, if $s=1$ then:

$$
\begin{gathered}
T_{1, p}(z)=\sum_{k=0}^{15} a_{k+1}(z+k d)^{p} \\
=\sum_{k=0}^{3} a_{k+1}(z+k d)^{p}+\sum_{k=4}^{7} a_{k+1}(z+k d)^{p} \\
+\sum_{k=8}^{11} a_{k+1}(z+k d)^{p}+\sum_{k=12}^{15} a_{k+1}(z+k d)^{p} \\
=T_{0, p}(z)+\sum_{m=0}^{3} a_{2^{2}+m+1}((z+4 d)+m d)^{p}
\end{gathered}
$$




$$
\begin{gathered}
+\sum_{m=0}^{3} a_{2^{3}+m+1}((z+2.4 d)+m d)^{p} \\
+\sum_{m=0}^{3} a_{2^{3}+2^{2}+m+1}((z+3.4 d)+m d)^{p} \\
=T_{0, p}(z)-\sum_{m=0}^{3} a_{m+1}((z+4 d)+m d)^{p} \\
-\sum_{m=0}^{3} a_{m+1}((z+2.4 d)+m d)^{p} \\
+\sum_{m=0}^{3} a_{m+1}((z+3.4 d)+m d)^{p} \\
=T_{0, p}(z)-T_{0, p}(z+4 d) \\
-T_{0, p}(z+2.4 d)+T_{0, p}(z+3.4 d) .
\end{gathered}
$$

The proof is similar in the general case:

$$
\begin{gathered}
T_{s, p}(z)=\sum_{k=0}^{4^{s+1}-1} a_{k+1}(z+k d)^{p} \\
=\sum_{k=0}^{4^{s}-1} a_{k+1}(z+k d)^{p}+\sum_{k=4^{s}}^{2.4^{s}-1} a_{k+1}(z+k d)^{p} \\
+\sum_{k=2.4^{s}}^{3.4^{s}-1} a_{k+1}(z+k d)^{p}+\sum_{k=3.4^{s}}^{4^{s+1}-1} a_{k+1}(z+k d)^{p} \\
=T_{s-1, p}(z)+\sum_{m=0}^{4^{s}-1} a_{4^{s}+m+1}\left(\left(z+4^{s} d\right)+m d\right)^{p} \\
+\sum_{m=0}^{4^{s}-1} a_{2.4^{s}+m+1}\left(\left(z+2.4^{s} d\right)+m d\right)^{p} \\
+\sum_{m=0}^{4^{s}-1} a_{3.4^{s}+m+1}\left(\left(z+3.4^{s} d\right)+m d\right)^{p} \\
=T_{s-1, p}\left(z+3.4^{s} d\right)-T_{s-1, p}\left(z+2.4^{s} d\right) \\
\quad-T_{s-1, p}\left(z+4^{s} d\right)+T_{s-1, p}(z),
\end{gathered}
$$

whence the necessary recurrent formula is established.

In the case $1 \leq s \leq\left[\frac{p}{2}\right]-1$, we prove that $T_{s, p}(z)$ has the type:

$$
\begin{gathered}
T_{s, p}(z)=\sum_{i_{1}=2 s}^{p-2} \sum_{i_{2}=2(s-1)}^{i_{1}-2} \sum_{i_{3}=2(s-2)}^{i_{2}-2} \cdots \\
\cdots \sum_{i_{s+1}=0}^{i_{s}-2}\left(\begin{array}{c}
p \\
i_{1}
\end{array}\right)\left(\begin{array}{c}
i_{1} \\
i_{2}
\end{array}\right)\left(\begin{array}{c}
i_{2} \\
i_{3}
\end{array}\right) \cdots\left(\begin{array}{c}
i_{s} \\
i_{s+1}
\end{array}\right) d^{p-i_{s+1}} L_{s, p} z^{i_{s+1}},
\end{gathered}
$$

where

$$
L_{s, p}=\left(3^{p-i_{1}}-2^{p-i_{1}}-1\right)\left(3^{i_{1}-i_{2}}-2^{i_{1}-i_{2}}-1\right) \cdots
$$

$$
\cdots\left(3^{i_{s}-i_{s+1}}-2^{i_{s}-i_{s+1}}-1\right) 4^{i_{1}+i_{2}+\cdots+i_{s}-s i_{s+1}} .
$$

Indeed, when $s=0$ follows:

$$
\begin{gathered}
T_{0, p}(z)=(z+3 d)^{p}-(z+2 d)^{p}-(z+d)^{p}+z^{p} \\
=\sum_{i_{1}=0}^{p-2}\left(\begin{array}{c}
p \\
i_{1}
\end{array}\right)\left(3^{p-i_{1}}-2^{p-i_{1}}-1\right) d^{p-i_{1}} z^{i_{1}} .
\end{gathered}
$$

Direct calculation for $T_{1, p}$ gives

$$
\begin{gathered}
T_{1, p}(z)=T_{0, p}(z)-T_{0, p}(z+4 d) \\
-T_{0, p}(z+2.4 d)+T_{0, p}(z+3.4 d) \\
=\sum_{i_{1}=0}^{p-2}\left(\begin{array}{c}
p \\
i_{1}
\end{array}\right)\left(3^{p-i_{1}}-2^{p-i_{1}}-1\right) d^{p-i_{1}} \\
\left((z+12 d)^{i_{1}}-(z+8 d)^{i_{1}}-(z+4 d)^{i_{1}}+z^{i_{1}}\right) \\
=\sum_{i_{1}=2}^{p-2}\left(\begin{array}{l}
p \\
i_{1}
\end{array}\right)\left(3^{p-i_{1}}-2^{p-i_{1}}-1\right) d^{p-i_{1}} \\
\left((z+12 d)^{i_{1}}-(z+8 d)^{i_{1}}-(z+4 d)^{i_{1}}+z^{i_{1}}\right) \\
=\sum_{i_{1}=2}^{p-2}\left(\begin{array}{l}
p \\
i_{1}
\end{array}\right)\left(3^{p-i_{1}}-2^{p-i_{1}}-1\right) d^{p-i_{1}} \\
\left(z^{i_{1}}+\sum_{i_{2}=0}^{i_{1}}\left(\begin{array}{l}
i_{1} \\
i_{2}
\end{array}\right)\left(3^{i_{1}-i_{2}}-2^{i_{1}-i_{2}}-1\right)(4 d)^{i_{1}-i_{2}} z^{i_{2}}\right) \\
=\sum_{i_{1}=2}^{p-2}\left(\begin{array}{l}
p \\
i_{1}
\end{array}\right)\left(3^{p-i_{1}}-2^{p-i_{1}}-1\right) d^{p-i_{1}} \\
\left.\left(\begin{array}{l}
i_{1}-2 \\
\sum_{i_{2}=0}^{i_{1}} \\
i_{2}
\end{array}\right)\left(3^{i_{1}-i_{2}}-2^{i_{1}-i_{2}}-1\right)(4 d)^{i_{1}-i_{2}} z^{i_{2}}\right)= \\
=\sum_{i_{1}=2}^{p-2} \sum_{i_{2}=0}^{i_{1}-2}\left(\begin{array}{l}
p \\
i_{1}
\end{array}\right)\left(\begin{array}{l}
i_{1} \\
i_{2}
\end{array}\right)\left(3^{p-i_{1}}-2^{p-i_{1}}-1\right) \\
\left(3^{i_{1}-i_{2}}-2^{i_{1}-i_{2}}-1\right) 4^{i_{1}-i_{2}} d^{p-i_{2}} z^{i_{2}}= \\
=\sum_{i_{1}=2}^{p-2} \sum_{i_{2}=0}^{i_{1}-2}\left(\begin{array}{l}
p \\
i_{1}
\end{array}\right)\left(\begin{array}{l}
i_{1} \\
i_{2}
\end{array}\right) d^{p-i_{2}} L_{1, p} z^{i_{2}}, \\
i_{1}
\end{gathered}
$$

hence the assertion is established for $s=1$. Suppose that for some $s \geq 2, T_{s-1, p}(z)$ satisfies the recurent formula and denote

$$
G_{i_{1}, i_{2}, \ldots, i_{s+1}}^{s, p}=\left(\begin{array}{c}
p \\
i_{1}
\end{array}\right)\left(\begin{array}{c}
i_{1} \\
i_{2}
\end{array}\right)\left(\begin{array}{c}
i_{2} \\
i_{3}
\end{array}\right) \cdots\left(\begin{array}{c}
i_{s} \\
i_{s+1}
\end{array}\right) d^{p-i_{s+1}} L_{s, p},
$$

for $s \geq 1$. Direct calculation shows:

$$
\begin{gathered}
T_{s, p}(z)=T_{s-1, p}\left(z+3.4^{s} d\right)-T_{s-1, p}\left(z+2.4^{s} d\right) \\
-T_{s-1, p}\left(z+4^{s} d\right)+T_{s-1, p}(z) \\
=\sum_{i_{1}=2(s-1)}^{p-2} \sum_{i_{2}=2(s-2)}^{i_{1}-2} \sum_{i_{3}=2(s-3)}^{i_{2}-2} \cdots \sum_{i_{s}=0}^{i_{s-1}-2} G_{i_{1}, i_{2}, \ldots, i_{s}}^{s-1, p}
\end{gathered}
$$




$$
\begin{aligned}
& \left(\left(z+3.4^{s} d\right)^{i_{s}}-\left(z+2.4^{s} d\right)^{i_{s}}-\left(z+4^{s} d\right)^{i_{s}}+z^{i_{s}}\right) \\
& =\sum_{i_{1}=2(s-1)}^{p-2} \sum_{i_{2}=2(s-2)}^{i_{1}-2} \cdots \sum_{i_{s}=0}^{i_{s-1}-2} G_{i_{1}, i_{2}, \ldots, i_{s}}^{s-1, p} \\
& {\left[z^{i_{s}}+\sum_{i_{s+1}=0}^{i_{s}}\left(\begin{array}{c}
i_{s} \\
i_{s+1}
\end{array}\right)\left(3^{i_{s}-i_{s+1}}-2^{i_{s}-i_{s+1}}-1\right)\right.} \\
& \left.4^{s\left(i_{s}-i_{s+1}\right)} d^{i_{s}-i_{s+1}} z^{i_{s+1}}\right] \\
& =\sum_{i_{1}=2 s}^{p-2} \sum_{i_{2}=2(s-1)}^{i_{1}-2} \cdots \sum_{i_{s}=2}^{i_{s-1}-2} G_{i_{1}, i_{2}, \ldots, i_{s}}^{s-1, p} \sum_{i_{s+1}=0}^{i_{s}-2} \\
& \left(\begin{array}{c}
i_{s} \\
i_{s+1}
\end{array}\right)\left(3^{i_{s}-i_{s+1}}-2^{i_{s}-i_{s+1}}-1\right) 4^{s\left(i_{s}-i_{s+1}\right)} d^{i_{s}-i_{s+1}} z^{i_{s+1}} \\
& =\sum_{i_{1}=2 s}^{p-2} \sum_{i_{2}=2(s-1)}^{i_{1}-2} \cdots \sum_{i_{s}=2}^{i_{s-1}-2} \sum_{i_{s+1}=0}^{i_{s}-2} G_{i_{1}, i_{2}, \ldots, i_{s}}^{s-1, p}\left(\begin{array}{c}
i_{s} \\
i_{s+1}
\end{array}\right) \\
& \left(3^{i_{s}-i_{s+1}}-2^{i_{s}-i_{s+1}}-1\right) 4^{s\left(i_{s}-i_{s+1}\right)} d^{i_{s}-i_{s+1}} z^{i_{s+1}} \\
& =\sum_{i_{1}=2 s}^{p-2} \sum_{i_{2}=2(s-1)}^{i_{1}-2} \cdots \sum_{i_{s}=2}^{i_{s-1}-2} \sum_{i_{s+1}=0}^{i_{s}-2} G_{i_{1}, i_{2}, \ldots, i_{s+1}}^{s, p} z^{i_{s+1}},
\end{aligned}
$$

which prove that $T_{s, p}(z)$ satisfies the recurrent formula.

Let us determine the degree of $T_{s, p}(z), s \geq 0$. According to the derived formula we find $i_{s+1} \leq i_{s}-2 \leq i_{s-1}-4 \leq \cdots \leq$ $i_{1}-2 s \leq p-2(s+1)$, as equality is reached everywhere. Therefore $\operatorname{deg} T_{s, p}(z)=p-2(s+1)$. If $p=2 m+r, r \in$ $\{0,1\}$, then

$$
\operatorname{deg} T_{m-1, p}(z)=p-2 m=r .
$$

For $r=0$ we obtain that $T_{m-1, p}(z)$ is a constant, equal to $p ! 2^{\frac{p^{2}-p}{2}} d^{p}$. Indeed

$$
\begin{gathered}
=\sum_{i_{1}=2(m-1)}^{p-2} \sum_{i_{2}=2(m-2)}^{i_{1}-2} \cdots \sum_{i_{m-1}=2}^{T_{m-1, p}(z)=} \sum_{i_{m}=0}^{i_{m-2}-2} G_{i_{1}, i_{2}, \ldots, i_{s+1}}^{m-1, p} z^{i_{m}} \\
=\sum_{i_{1}=2(m-1)}^{2(m-1)} \sum_{i_{2}=2(m-2)}^{2(m-2)} \cdots \sum_{i_{m-1}=2}^{2} \sum_{i_{m}=0}^{0} \\
G_{i_{1}, i_{2}, \ldots, i_{s+1}}^{m-1, p} z^{i_{m}} \\
=G_{p-2, p-4, p-6 \ldots, 2,0}^{m-1, p} \\
=\left(\begin{array}{c}
p \\
p-2
\end{array}\right)\left(\begin{array}{l}
p-2 \\
p-4
\end{array}\right) \cdots\left(\begin{array}{l}
4 \\
2
\end{array}\right)\left(\begin{array}{l}
2 \\
0
\end{array}\right) d^{p} L_{m-1, p}
\end{gathered}
$$

$$
\begin{gathered}
=\frac{p ! d^{p}}{2^{m}} L_{m-1, p}=p ! 2^{\frac{p^{2}-p}{2}} d^{p} \Longrightarrow \\
\Longrightarrow T_{m-1, p}(z)=p ! 2^{\frac{p^{2}-p}{2}} d^{p}, \text { for } p=2 m .
\end{gathered}
$$

In the case $r=1$, we will prove that $T_{m, p}(z)=0$ :

$$
\begin{gathered}
T_{m, p}(z)= \\
=T_{m-1, p}\left(z+3.4^{m} d\right)-T_{m-1, p}\left(z+2.4^{m} d\right)- \\
T_{m-1, p}\left(z+4^{m} d\right)+T_{m-1, p}(z) \\
=\sum_{i_{1}=2(m-1)}^{p-2} \sum_{i_{2}=2(m-2)}^{i_{1}-2} \cdots \sum_{i_{m}=0}^{i_{m-1}-2} G_{i_{1}, i_{2}, \ldots, i_{m}}^{m-1, p} \\
\left(\left(z+3.4^{m} d\right)^{i_{m}}-\left(z+2.4^{m} d\right)^{i_{m}}-\left(z+4^{m} d\right)^{i_{m}}+z^{i_{m}}\right)=0,
\end{gathered}
$$

and the above equality is valid, since the summation index $i_{m}$ takes values 0 and 1 . Thus the proposition 6 is proved. Proofs of propositions 1,2,3,4,5 will be presented in [7].

\section{ACKNOWLEDGEMENT}

Venelin Todorov is supported by the Bulgarian National Science Fund under Project DN 12/5-2017 "Efficient Stochastic Methods and Algorithms for Large-Scale Problems" and by the National Scientific Program "Information and Communication Technologies for a Single Digital Market in Science, Education and Security" (ICTinSES), contract No. D01-205/23.11.2018, financed by the Ministry of Education and Science. Miroslav Stoenchev is supported by KP-06M32/2-17.12.2019 "Advanced Stochastic and Deterministic Approaches for Large-Scale Problems of Computational Mathematics".

\section{REFERENCES}

[1] J. -P. Allouche, J. O. Shallit, The ubiquitous Prouhet-Thue-Morse sequence. In C. Ding, T. Helleseth, and H. Niederreiter, editors, Sequences and Their Applications, Proceedings of SETA '98, pp. 1-16, SpringerVerlag, 1999.

[2] P. Borwein, C. Ingalls, The Prouhet-Tarry-Escott Problem revisited. Enseign. Math. 40 (1994), 3-27. MR 95d:11038

[3] P. Borwein, Computational Excursions in Analysis and Number Theory, Springer-Verlag, New York, 2002.

[4] J. Byszewski and M. Ulas, Some identitites involving the Prouhet-ThueMorse sequence and its relatives, Acta Math. Hungar., 127-2,438-456, 2015

[5] H.L. Dorwart and O.E. Brown, The Tarry-Escott Problem, M.A.A. Monthly, 44, 613-626, 1937

[6] G.H. Hardy, E.M. Wright, An Introduction to the Theory of Numbers, fifth ed., Oxford Univ. Press, New York,1979

[7] M. Stoenchev, V. Todorov, Combinatorial etudes and number theory, (in appear)

[8] T. Wakhare, C. Vignat, Settling some sum suppositions, arXiv:1805.10569 [math.NT] 